\title{
Labyrinthe
}

$15 \mid 2003$

Territoires : questions d'images

\section{Le Prix étudiant du livre politique 2003}

\section{(2) OpenEdition}

1 Journals

Édition électronique

URL : http://journals.openedition.org/labyrinthe/490

DOI : $10.4000 /$ labyrinthe.490

ISSN : 1950-6031

Éditeur

Hermann

Édition imprimée

Date de publication : 1 juin 2003

Référence électronique

"Le Prix étudiant du livre politique 2003 », Labyrinthe [En ligne], 15 | 2003, mis en ligne le 25 juillet 2008, consulté le 21 avril 2019. URL : http://journals.openedition.org/labyrinthe/490 ; DOI : 10.4000/ labyrinthe. 490

Ce document a été généré automatiquement le 21 avril 2019.

Propriété intellectuelle 


\section{Le Prix étudiant du livre politique 2003}

1 LABYRINTHE, partenaire du Prix étudiant du Livre politique, décerné le 29 mars 2003 à l'Assemblée nationale, à l'occasion de la $12^{\mathrm{e}}$ Journée du Livre politique.

2 Créé en 1998, le Prix étudiant du livre politique entend développer l'intérêt des jeunes auteurs pour les sujets politiques. Pour la deuxième année consécutive, le Prix a été attribué au manuscrit non publié d'un étudiant, afin de favoriser l'écriture et d'encourager de nouveaux talents.

3 Un jury d'étudiants composé de rédacteurs du réseau des "Revues indépendantes » récompense, parmi des manuscrits reçus sur le site web de manuscrit.com, un texte traitant d'un sujet politique, au sens large (histoire, philosophie politique, géopolitique, science politique, droit, sociologie, etc.).

4 Le manuscrit lauréat, désigné par le jury des «Revues indépendantes » est publié par les Éditions Nicolas Philippe et se voit ainsi assuré d'une diffusion en librairie. Le premier tirage est de 3000 exemplaires.

5 Avec le parrainage d'Anima'fac, réseau national d'associations étudiantes, fondateur du prix, et dans la lignée du Prix du Livre Politique, Lire la politique, manuscrit.com/ Éditions Nicolas Philippe, Les revues indépendantes, et Le Mouv' souhaitent, par cette initiative, donner toute sa force à l'analyse, au discours politique et renouer avec les vertus des valeurs républicaines.

\section{Le jury 2003}

6 Présidé par Noe DA SILVA, journaliste au Mouv', le jury étudiant est composé des rédacteurs en chef des revues membre du réseau des Revues indépendantes.

7 Président du jury :

Noé DA SILVA, journaliste au Mouv'

8 Secrétaire général du Prix étudiant du Livre politique :

François-Xavier Priollaud, revue Labyrinthe

9 Les membres du jury :

Jean-Noël BIGOTTI, revue Copyright Volume!; Cécile-Fleur BRUNOD, revue Le Crochet de la 
cédille; Lucas DEGRYSE, revue Le Philosophoire; Isabelle DUMOULIN, revue Idées ; François GREMY, revue Regard sur l'Est ; Laurence MARIE, revue Labyrinthe; John PALACIN, revue Parages ; Mathieu ROUAULT, revue Le Mensuel ;

Nicolas VIMAR, revue Immédiatement.

\section{La lauréate 2003} un leitmoviv, selon laquelle «pour une majorité de français, droite et gauche ne veulent plus rien dire». A mieux étudier ces sondages, on s'aperçoit que ce qui est disqualifié c'est l'absence de visibilité des différences objectives entre les grands partis qui rythment la vie politique française, bien plus qu'une remise en cause de ses repères structurants. Ce qui est critiqué, c'est par exemple, que la gauche ne soit plus assez à gauche - en bref, on continue de penser dans une logique d'antagonisme !

SYLVIE AEBISCHER, 22 ANS, Droite, gauche : au-delà de cette limite, la politique n'est plus pensable, Ed. Nicolas Philippe, 131 p.,16 euros.

Diplômée de l'Institut d'études politiques de Lyon, Sylvie Aebischer a soutenu son mémoire «Le clivage droite-gauche comme ressource structurante des perceptions politiques françaises », sous la direction de M. Jean-Louis Marie, dans le cadre d'un DEA de science politique à l'université Lyon II. Elle prépare actuellement une thèse de science politique (au laboratoire d'accueil CERIEP - Centre de politologie de Lyon) et est chargée de cours à l'IEP de Lyon et à la faculté de droit de Lyon II.

\section{Entretien avec Sylvie Aebischer}

\section{Pourquoi avoir participé au Prix étudiant du livre politique?}

Sylvie AEBISCHER - C'est un ami qui avait entendu parler du concours, qui m'en a parlé. Il m'a dit: " toi qui a obtenu une bonne note à ton mémoire de DEA, tu devrais l'envoyer ! ». J'ai suivi son conseil, sans imaginer un instant pouvoir gagner... On a parfois de bonnes surprises!

\section{Le clivage gauche / droite : pourquoi avoir choisi ce sujet?}

Sylvie AEBISCHER - Parce que je n'arrivais pas à me faire comprendre des allemands lorsque je parlais politique... Les allemands n'utilisent pas aussi abondamment que nous ces mots, ce qui m'a posé un problème de vocabulaire... il n'en demeure pas moins que leur représentation du monde politique est tout aussi structurée. Avec l'Europe nous aurons peut-être un changement de vocabulaire: mais je ne crois pas que nous soyons dans la capacité de penser autrement qu'à partir de catégories duales.

\section{Que pensez-vous de l'avenir du clivage droite-gauche aujourd'hui en France et en} Europe?

Sylvie AEBISCHER - Mon travail se comprend, je crois, d'abord comme une «critique d'une mort annoncée »... On parlait déjà de la droite et de la gauche comme de notions archaïques et dépassées dans les années 20. On parle un peu trop vite lorsqu'on enterre ces notions sans réfléchir aux mots avec lesquels les gens continuent de penser la politique. Or nous pensons en mode binaire, et il est difficile de dépasser ses propres structures de pensée. Ce que j'ai essayé de montrer est qu'il ne faut pas réduire le clivage 
droite-gauche à une opposition de valeurs figées, et qu'il s'agit de termes efficaces pour organiser une vision du monde. En pensant en termes de droite et gauche, nous projetons sur la vie politique et sociale une interprétation binaire des choses : le clivage droitegauche est une sorte de raccourci qui nous permet de traduire simplement la complexité du monde. Chacun se forge une compréhension personnalisée de ces notions en fonction de ses valeurs de référence, mais il ne la fait pas ex nihilo, et on observe forcément une certaine sédimentation culturelle $\mathrm{du}$ contenu des notions.L'opposition la plus représentative aujourd'hui du clivage droite / gauche est difficile à exprimer, la gauche de Chevènement n'est pas celle de Besancenot, ni celle de Jospin. On peut tout de même avancer que pour quelqu'un de gauche elle correspondra généralement à l'opposition entre politiques sociales et libérales. Quelqu'un de droite la traduira plutôt en termes de sens de l'effort et de la responsabilité au regard d'une logique d'assistanat. Dans les deux cas, on observe une stigmatisation de l'autre et dans une certaine mesure, on peut dire que le clivage droite-gauche recoupe l'asymétrie Moi / Autrui sur le modèle de l'opposition entre le Bien et le Mal.

\section{engagements différents?}

Sylvie AEBISCHER - Je pense, comme le disait Bourdieu, que « la jeunesse n'est qu'un mot », qu'elle n'existe pas en tant que catégorie homogène. On dit souvent que les jeunes développent de nouvelles valeurs, en rupture avec celles de leurs aînés. Selon moi, ils en sont incapables précisément parce qu'ils ne constituent pas un groupe en soi. Certes, les manifestations anti-FN ne se sont pas faites au nom de la gauche, mais dans une logique de définition de soi-même par opposition à un idéal rebutant - un clivage droite-gauche en puissance qui certainement n'attend que la première occasion pour éclore. Propos recueillis par François-Xavier Priollaud, Secrétaire général du Prix étudiant du livre politique 